\title{
Application of the Construction of Food Safety Network Platform in Diabetes Management
}

\author{
Chih Chiang Chao*, Yih Ming Weng \\ Research Institute, Department of Food Science, National Chiayi University, Taiwan.
}

How to cite this paper: Chih Chiang Chao, Yih Ming Weng. (2021) Application of the Construction of Food Safety Network Platform in Diabetes Management. International Journal of Clinical and Experimental Medicine Research, 5(2), 135-138.

DOI: 10.26855/ijcemr.2021.04.007

Received: February 6, 2021

Accepted: March 2, 2021

Published: March 24, 2021

*Corresponding author: Chih Chiang Chao, Research Institute, Department of Food Science, National Chiayi University, Taiwan.

\begin{abstract}
Objective: To explore and analyze the application of the construction of a food safety network platform in diabetes management. Methods: A total of 90 patients with diabetes management who were admitted to our hospital from May 2018 to May 2020 were selected and divided into the routine management group and the network platform management group by random number table, 45 cases in each group, and the comparison between the two groups Diabetes related health knowledge awareness rate, healthy diet compliance rate and blood sugar control rate. Results: Compared with the routine management group, patients in the online platform management group had higher awareness rates of diabetes-related food knowledge, healthy diet compliance and blood sugar control rates, and the difference was statistically significant $(\mathrm{P}<0.05)$. Conclusion: The construction of a food safety network platform and its application in diabetes management can significantly improve patients' awareness of diabetes-related food knowledge, and at the same time improve patients' compliance with healthy diets and improve the effect of blood sugar control.
\end{abstract}

\section{Keywords}

Food Safety, Network Platform, Diabetes Management

Diabetes is a chronic disease with a high clinical incidence. Hyperglycemia is the main pathological feature. It is caused by relative or absolute lack of insulin and different degrees of insulin resistance. In severe cases, different degrees of carbohydrates and fats may appear. Other disorders, and more complications, have a greater impact on the quality of life of patients [1]. In recent years, with the continuous deepening of diabetes research, it has been found that diabetes also has a certain degree of food safety problems. Reports point out that Monosodium Glutamate (MSG) intake is positively correlated with insulin resistance, which will increase the risk of diabetes [2]. In addition, excessive absorption of phosphorus is especially important for diabetic patients, especially those with nephropathy, when renal excretion decreases, and it can cause acute phosphate nephropathy in severe cases. Therefore, it is very important to strengthen the food safety management of diabetes [3]. This research combines modern network technology to build a food safety network platform and apply it to diabetes management, aiming to achieve better results. The results are reported as follows.

\section{Materials and methods}

\subsection{General information}

A total of 90 patients undergoing diabetes management who were admitted to our hospital from May 2018 to May 2020 were selected and divided into routine management group and network platform management group by random number table, 45 cases in each group, 25 male cases in the routine management group, 21 female cases, 
aged 50 to 73 years, average (65.43 \pm 4.11$)$ years, course of illness 4 to 10 years, average $(7.78 \pm 1.02)$ years. In the online platform management group, there were 26 males and 19 females, aged 52 to 71 years old, with an average of (65.20 \pm 4.28$)$ years old, and the course of disease was 2 to 9 years, with an average of $(7.25 \pm 1.23)$ years. There is no significant difference in general information between the two groups and they are comparable.

\subsection{Method}

The routine management group gives basic diabetes management methods, including medication management, monitoring indicators, and health education. On the basis of the conventional management team, the network platform management team builds and applies the food safety network platform.

Method: After the patient is discharged from the hospital, a detailed electronic file is established and the electronic file is incorporated into the basic information. The doctor combines the patient's information Develop a reliable food safety and diet plan for the specific situation.

Through the call center platform, we can provide patients with active call services on a regular basis. Compared with the tracking and guidance of the entire diet plan during the patient's stay at home, the patient can also be guided when needed. Under the premise of help, dial the designated telephone number to call the network platform service center, and the professional nurses will understand the individual information of the patient and give professional answers [4] in conjunction with the phone numbers of the patients.

In addition, the food safety network platform can input what you have eaten today and how many grams you weigh. You can calculate from the mobile phone program whether the diet exceeds the limit and whether the content of food additives is toxic. It can also detect the additives of their products from all qualified manufacturers who have found the additives in the food from the local health or private units of the product. Information is obtained from food manufacturers, primary wholesalers, secondary distributors, and retail sales.

\subsection{Observation indicators}

Compare the two groups of patients' awareness rate of diabetes-related food knowledge, healthy diet compliance rate and blood sugar control rate.

\subsection{Statistical processing}

Use SPSS18.0 statistical software to analyze the data. The enumeration data is expressed in \%, using the $\chi^{2}$ test, and $\mathrm{P}<0.05$ represents statistical significance.

\section{Results}

\subsection{Comparison of the awareness rate of diabetes-related food knowledge, healthy diet compliance rate and blood sugar control rate between the two groups}

Compared with the routine management group, the patients' awareness rate of diabetes-related food knowledge, healthy diet compliance rate and blood sugar control rate were higher in the online platform management group and the difference was statistically significant $(\mathrm{P}<0.05)$ (see Table 1 ).

Table 1. Comparison of the awareness rate of diabetes-related food knowledge, healthy diet compliance rate and blood sugar control rate between the two groups of patients [case (\%)]

\begin{tabular}{ccccc}
\hline Group & $\begin{array}{c}\text { Number of } \\
\text { cases }\end{array}$ & $\begin{array}{c}\text { Awareness rate of di- } \\
\text { abetes-related food knowledge }\end{array}$ & $\begin{array}{c}\text { Healthy diet } \\
\text { compliance rate }\end{array}$ & $\begin{array}{c}\text { Blood glucose } \\
\text { control rate }\end{array}$ \\
\hline $\begin{array}{c}\text { Routine Management } \\
\text { Group }\end{array}$ & 45 & $33(73.33)$ & $31(68.89)$ & $32(71.11)$ \\
$\begin{array}{c}\text { Network platform man- } \\
\text { agement group }\end{array}$ & 45 & $42(93.33)$ & $41(91.11)$ & $43(95.56)$ \\
$\chi^{2}$ & & 6.480 & 6.944 & 9.680 \\
$P$ & 0.011 & 0.008 & 0.002 \\
\hline
\end{tabular}

\section{Discussion}

Diabetes is a chronic disease. Due to relative or absolute lack of insulin and varying degrees of insulin resistance, 
it causes carbohydrate, fat and protein metabolism disorders, and is a comprehensive disease with persistent high blood sugar as its basic biochemical characteristics. Controlling the safety and quality of eating is the basic measure for the treatment of diabetes [5]. Regardless of whether it is light or heavy, whether it is insulin or oral medication, diet control must be used to reduce the burden of pancreatic beta cells and improve symptoms.

For elderly, obese and mild cases with asymptomatic or few symptoms, especially those with high levels of plasma insulin during fasting and after meals, nutritional control is often the first measure. For severe cases, in addition to drug treatment, it is more appropriate to strictly control diet safety to prevent fluctuations in the condition.

Food safety is an interdisciplinary field that specifically discusses how all parties can ensure food hygiene and food safety, reduce hidden diseases and prevent food poisoning in the steps of food processing, storage, and sales. Explore the risk analysis of items that may cause consumer health hazards through scientific methods, and then formulate control measures to ensure food safety.

Using food safety measures to eliminate or reduce risks to consumers' lives and health is the core of food safety. In a broad sense, food safety also includes a reasonable and balanced diet [6].

However, in recent years, many food safety issues have been discovered in recent years. Because three meals a day are inseparable from eating, the public pays more attention to the safety of what you eat; there is another reason why food safety is continuously concerned. The consequences are serious, and are often associated with poison and cancer.

Chronic diseases such as heart disease, cancer, cerebrovascular disease and diabetes are becoming more common, and food safety issues are getting more and more serious, which may also lead to an increase in mortality. Diet-related cancers accounted for $30 \%$. Pickled foods are related to gastric cancer, colorectal cancer and nasopharyngeal cancer. Food additives may increase the risk of bladder cancer. Food contamination caused by aflatoxin can cause liver cancer, etc. [7]. There is a positive correlation between MSG intake and insulin resistance, which can increase the risk of diabetes. Trans fatty acids are also closely related to the occurrence of diabetes, Alzheimer's disease and certain cancers.

Research data show that the association between diabetes and infection is very common. Even minor infections can interfere with blood sugar control [8]. In addition to common community diseases, certain gastrointestinal infections and food poisoning caused by unsafe food may mainly occur in diabetic patients, especially in the case of insufficient blood sugar control, the common infections of these patients may be more serious [9].

The disease itself and changes in immune response caused by unsafe food are responsible for the higher frequency and risk of serious infections. During infection and poisoning, blood sugar will increase, and insulin doses are usually increased. Unsafe food and food poisoning will in turn increase the risk of acute metabolic decompensation in diabetic patients during infection or illness, while patients with metabolic decompensation are more likely to suffer other aggressive infections [10] .

In order to better improve food safety, this research has built and applied a food safety network platform. By establishing a network platform that can directly connect patients with medical workers, it can not only be effective on patients' diets after discharge. At the same time, patients can provide professional answers at any time when they face effective questions. In addition, the application of the food safety network platform can help patients obtain effective food-related safety management information on the one hand, and can calculate from the mobile phone program whether the diet exceeds the limit and whether the content of food additives is toxic, which can be better improved.

Improve the quality of food safety management for diabetic patients, so as to obtain better intervention effects. In future research, the work content of diabetes can be continuously enriched in light of the specific conditions of diabetes, such as focusing on promoting the traceability system of edible agricultural products and actively exploring the safety certification of agricultural products, so as to improve the awareness of agricultural product quality and safety by using the food safety certification system.

The results show that compared with the conventional management group, the network platform management group has a higher awareness rate of diabetes-related food knowledge, healthy diet compliance rate and blood sugar control rate among patients, suggesting that the construction and application of a food safety network platform can significantly improve food Safety management quality, so as to better help patients understand and clarify the patient's awareness rate of diabetes-related food knowledge, and at the same time improve their compliance with healthy diets, reduce the adverse effects on blood sugar levels, and obtain higher blood sugar Control rate.

In summary, the construction of a food safety network platform and its application in diabetes management can significantly improve patients' awareness of diabetes-related food knowledge, and at the same time improve patients' compliance with healthy diets, and improve blood sugar control effects. In future research, the research sam- 
ple size can be further expanded, and the follow-up time can be extended to better construct and apply the food safety network platform.

\section{References}

[1] Jaclyn Tamaroff, Marissa Kilberg. (2020). Overview of Atypical Diabetes. Endocrinology and Metabolism Clinics of North America, 2020, 49: 695-723.

[2] F. J. Carrasco-Sánchez, M. Fernández-Rodríguez. (2020). Medical treatment of type 2 diabetes mellitus: Recommendations of the Diabetes, Obesity and Nutrition Group of the Spanish Society of Internal Medicine. Revista Clínica Española (English Edition), 2020, 14: 123.

[3] KDIGO 2020 Clinical Practice Guideline for Diabetes Management in Chronic Kidney Disease. Kidney International 2020.

[4] Morgan Hampton Randall, Zachary Merle Haulsee, Jingwen Zhang. (2020). The effect of remote patient monitoring on the primary care clinic visit frequency among adults with type 2 diabetes. International Journal of Medical Informatics, 2020, 143: 104267.

[5] Renata B. Levy, Fernanda Rauber, Kiara Chang. (2020). Ultra-processed food consumption and type 2 diabetes incidence: A prospective cohort study. Clinical Nutrition, 2020, 12: 18.

[6] Ehab S. Eshak, Isao Muraki, Hironori Imano. (2021). Manganese intake from foods and beverages is associated with a reduced risk of type 2 diabetes. Maturitas, 2021, 143: 127-131.

[7] Monica Galloa, Lydia Ferrarab, Armando Calogero. (2020). Relationships between food and diseases: What to know to ensure food safety. Food Research International, 2020, 137: 109414.

[8] Yu-Ju Chu, Hwai-I Yang, Hui-Chen Wu. (2018). Aflatoxin B1 exposure increases the risk of hepatocellular carcinoma associated with hepatitis C virus infection or alcohol consumption. European Journal of Cancer, 2018, 94: 37-46.

[9] Tamara Turk Wensveen, Dora Gasparini, Dario Rahelic. (2021). Type 2 diabetes and viral infection; cause and effect of disease. Diabetes Research and Clinical Practice, 2021, 172: 108637.

[10] Yong-ning Wu, Jun-shi Chen. (2018). Food safety monitoring and surveillance in China: Past, present and future. Food Control, 2018, 90: 429-439. 